\title{
New analysis of the two carbon-rich stars CS 22948-27 and CS 29497-34: Binarity and neutron capture elements ${ }^{\star}$
}

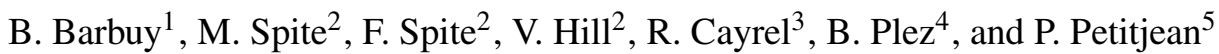 \\ 1 Universidade de São Paulo, Rua do Matão 1226, São Paulo 05508-900, Brazil \\ e-mail: barbuy@astro.iag.usp.br \\ 2 Observatoire de Paris-Meudon, GEPI, URM 8633 du CNRS, 92195 Meudon Cedex, France \\ 3 Observatoire de Paris, GEPI, 61 Av. de l'Observatoire, 75014 Paris, France \\ 4 GRAAL cc72, Université de Montpellier II, 34095 Montpellier Cedex 5, France \\ 5 Institut d'Astrophysique, 98bis Bd. Arago, 75014 Paris, France
}

Received 9 March 2004 / Accepted 27 August 2004

\begin{abstract}
We have carried out a new determination of abundances in the very metal-poor CH/CN strong stars CS 22948-27 and CS 29497-34, using high-resolution spectra obtained with the HARPS spectrograph at the $3.6 \mathrm{~m}$ telescope of ESO, La Silla, that covers the range $\lambda \lambda 4000-6900 \AA$ at a resolution of $R=100000$. Both stars are found to be long period binaries. It is confirmed that the abundance patterns show an enhancement of the $\alpha$-elements (like $\mathrm{Mg}, \mathrm{Ca}$ ), of the proton capture elements (like $\mathrm{Na}$ and $\mathrm{Al}$ ) and a strong enrichment in " $\mathrm{r}$ " and "s" process elements, where the s-enrichment is probably due to a mass transfer episode from a companion in its AGB phase. The possible origins of the abundance pattern and especially of the strong enhancement of both "s" and " $r$ " elements are discussed.
\end{abstract}

Key words. stars: abundances - stars: population II - stars: carbon - stars: binaries: spectroscopic - Galaxy: bulge nuclear reactions, nucleosynthesis, abundances

\section{Introduction.}

The most metal-poor stars in the Galaxy show an unexpected fraction of carbon-rich stars, as first pointed out by Beers et al. (1992), Norris et al. (1997), Rossi et al. (1999). More recently, a deeper survey indicated (Christlieb 2003) that about 30\% of the most metal-poor stars are carbon enhanced. These stars are often enriched also in nitrogen and in neutron capture heavy elements. The enhancement of s-process and/or r-process elements varies however from star to star.

The enrichment in neutron-capture $s$-elements suggests either an intrinsic enrichment in the star itself (post-AGB or helium flash), or an extrinsic process, e.g. by mass transfer from a companion in its Asymptotic Giant Branch (AGB) phase.

An analysis of the elemental abundances of the carbon stars BPS CS 22948-27 and BPS CS 29497-34 (Barbuy et al. 1997; Hill et al. 2000, Paper I) has shown that these stars are rich in $s$ and $r$-elements. Only few very metal-poor stars are known with a strong "r" and "s" enrichment (Zijlstra 2004; Cohen et al. 2003, hereafter CC03). In our previous work we were not able to determine the abundance of lead which is a key element to

* Observations collected at the European Southern Observatory (ESO), ESO Programme 72.A-0244 (PI Petitjean), and including data obtained from the ESO/ST-ECF Science Archive Facility. determine the neutron exposure since it belongs to the "third peak" of the "s" elements.

Recently new high resolution spectra $(R=100000)$ of CS 22948-27 and CS 29497-34 have been obtained at ESO with the high resolution spectrograph HARPS. In these spectra, several lines appear now less severely blended and the accuracy of the abundance determination is much improved. In this paper we redetermine the abundances of the elements (and in particular those of $\mathrm{Eu}$ and $\mathrm{Pb}$ ), based on these new very high resolution spectra. The opacities and models computed for these peculiar chemical compositions are the same as those used in the previous paper, whereas revised line lists of $\mathrm{CH}$ and $\mathrm{CN}$ blue, and hyperfine structure for the heavy elements are taken into account.

In order to better understand the cause of the overabundance of the heavy elements in carbon stars it is important to know whether they are binaries. In this case, an enhancement of the "s" elements for example can be explained by a transfer of matter from an AGB companion. In Hill et al. (2000), from observations in the period 1995-1999, we could not detect any clear variation of the radial velocity of CS 22948-27 and CS 29497-34. However, the radial velocity of CS 22948-27 has been found recently to be variable by Preston \& Sneden (2001). Using our additional new data, we have found that the radial 
velocity of CS 29497-34 is also variable and we have made a new determination of the orbital parameters of the two stars.

In Sect. 2 the observations and reduction procedures are reported and new orbital elements for CS 22948-27 and CS 29497-34 are determined. In Sect. 3 the spectrum synthesis calculations and abundance determinations are described, and the results are presented. In Sect. 4 these results are discussed and compared to other analyses of heavy element enriched carbon-stars, in order to try to find possible scenarios for the formation of these peculiar stars. Finally, concluding remarks are given in Sect. 5 .

\section{Observations}

New high resolution spectra were obtained for CS 22948-27 and CS 29497-34, in the wavelength region 3800-6900 A, using the $3.6 \mathrm{~m}$ telescope at ESO, equipped with the High Accuracy Planetary Search Spectrograph (HARPS). The resolution of these spectra is very high $(R=100000)$ and their $S / N$ ratio per pixel (difficult to estimate because of the crowded lines) is about 50 at $4000 \AA$ and 100 at $6000 \AA$. Although they have a moderate $S / N$ ratio, their high resolution is a significant advantage for the identification and measurement of the lines in these very crowdy spectra. The HARPS spectra have been reduced by the corresponding pipeline at the telescope (division by a flat-field frame, optimal extraction of the orders, wavelength calibration, and merging of all overlapping orders).

A spectrum of CS 29497-34 from the ESO archive obtained with UVES at the VLT-UT2 telescope has also been used for radial velocity measurements. This spectrum has a resolution of 45000 , and the spectral coverage is $3600-4800 \AA$ in the blue and 5880-7400 $\AA$ in the red. This archive UVES spectrum has been reduced using the UVES context (Ballester et al. 2000) within MIDAS, and its $S / N$ ratio is close to 200 .

The log of the new observations is reported in Table 1 (UVES and HARPS spectra). In this table we included also our old less precise observations of these stars from the ESO spectrographs EMMI, CASPEC and FEROS (see Hill et al. 2000) for the determination of the orbital parameters.

\subsection{Radial velocities and orbital elements}

From the new high resolution spectra obtained at UVES and HARPS we have derived precise measurements of the radial velocity of CS 22948-27 and CS 29497-34 in 2002, 2003 and 2004. The barycentric radial velocities determined from the position of the iron lines are given for all the spectra in Table 1. It is difficult to find unblended lines in these cool stars and the formal standard deviation of the mean radial velocity is 0.27 and $0.28 \mathrm{~km} \mathrm{~s}^{-1}$ for the HARPS and UVES spectra respectively.

\subsubsection{CS $22948-27$}

Recently Preston \& Sneden (2001) have monitored the radial velocity of CS 22948-27 at Las Campanas observatory and found it variable with a period of 501 days, but the data were sparse and the period uncertain. Our measurement
Table 1. Log-book of the observations and Radial Velocities measurements.

\begin{tabular}{ccccc}
\hline \hline & Julian d. & exp. & $\lambda$ & $V r_{\text {bary }}$ \\
$\mathrm{R}$ & $-2.4 \times 10^{6} \mathrm{~d}$ & $(\min )$ & $(\mathrm{nm})$ & $\mathrm{km} \mathrm{s}^{-1}$ \\
\hline
\end{tabular}

CS 22948-27

EMMI

30000

$\begin{array}{lccc}49976 . & 120 & 500-820 & -63.9 \pm 1.5 \\ 49978 . & 105 & 500-820 & -64.7 \pm 1.5 \\ 50025 . & 60 & 500-820 & -62.3 \pm 1.5 \\ 50026 . & 60 & 500-820 & -62.7 \pm 1.5 \\ 50027 . & 55 & 500-820 & -62.8 \pm 1.5\end{array}$

CASP.

21000

$\begin{array}{lccc}50358 . & 90 & 400-500 & -60.6 \pm 3.0 \\ 50359 . & 120 & 400-500 & -67.3 \pm 3.0\end{array}$

FEROS

48000

$\begin{array}{llll}51575 . & 30 & 400-850 & -67.1 \pm 1.0 \\ 51384 . & 30 & 400-850 & -67.4 \pm 1.0 \\ 51394 . & 30 & 400-850 & -68.1 \pm 1.0\end{array}$

HARPS

\begin{tabular}{lcccc}
100000 & 53142. & 80 & $380-690$ & $-69.9 \pm 0.3$ \\
\hline
\end{tabular}

CS 29497-34

EMMI

30000

$\begin{array}{llll}49975 . & 120 & 500-820 & -44.6 \pm 1.5 \\ 49975 . & 95 & 500-820 & -44.7 \pm 1.5 \\ 50025 . & 90 & 500-820 & -46.6 \pm 1.5 \\ 50026 . & 75 & 500-820 & -45.7 \pm 1.5\end{array}$

CASP.

\begin{tabular}{lllll}
21000 & 50358. & 120 & $400-500$ & $-43.3 \pm 3.0$ \\
& 50359. & 120 & $400-500$ & $-42.2 \pm 3.0$ \\
FEROS & & & & \\
48000 & 51575. & 30 & $400-850$ & $-44.2 \pm 1.0$ \\
& 51384. & 30 & $400-850$ & $-44.5 \pm 1.0$ \\
& 51394. & 30 & $400-850$ & $-44.7 \pm 1.0$ \\
UVES & & & & \\
45000 & 52472. & 45 & $480-680$ & $-51.4 \pm 0.5$ \\
HARPS & & & & \\
100000 & 52994. & 180 & $380-690$ & $-52.7 \pm 0.3$ \\
\hline
\end{tabular}

of the radial velocity in 2004 cannot be reconciled with the curve of the orbital variations of the radial velocity given by Preston and Sneden (at an orbital phase $\phi=0.96$ we find $R V=-69.9 \pm 0.3 \mathrm{~km} \mathrm{~s}^{-1}$, whereas from the published curve we would expect $-65.0 \mathrm{~km} \mathrm{~s}^{-1}$ ). However, in the determination of the orbital parameters Preston \& Sneden (2001) had rejected two Las Campanas observations (JD $50997 R V=$ -69.8 and JD $\left.51034 R V=-72.1 \mathrm{~km} \mathrm{~s}^{-1}\right)$. We supplemented our own ESO observations of CS22948-27 with all the Las Campanas Observatory data. We have replaced our two imprecise CASPEC measurements (see Table 1) by their mean 
Table 2. Provisional orbital elements for CS 22948-27 and CS 29497-34.

\begin{tabular}{lcc}
\hline \hline Element & CS 22948-27 & CS 29497-34 \\
\hline JDo & 2448110 & 2449800 \\
$\gamma\left(\mathrm{km} \mathrm{s}^{-1}\right)$ & -67.5 & -47.5 \\
$\mathrm{~K} 1\left(\mathrm{~km} \mathrm{~s}^{-1}\right)$ & 4.4 & 5.2 \\
$e$ & 0.02 & 0.02 \\
$\omega(\mathrm{rad})$ & 0.011 & 0.07 \\
$P($ days $)$ & 426.5 & 4130 \\
\hline
\end{tabular}

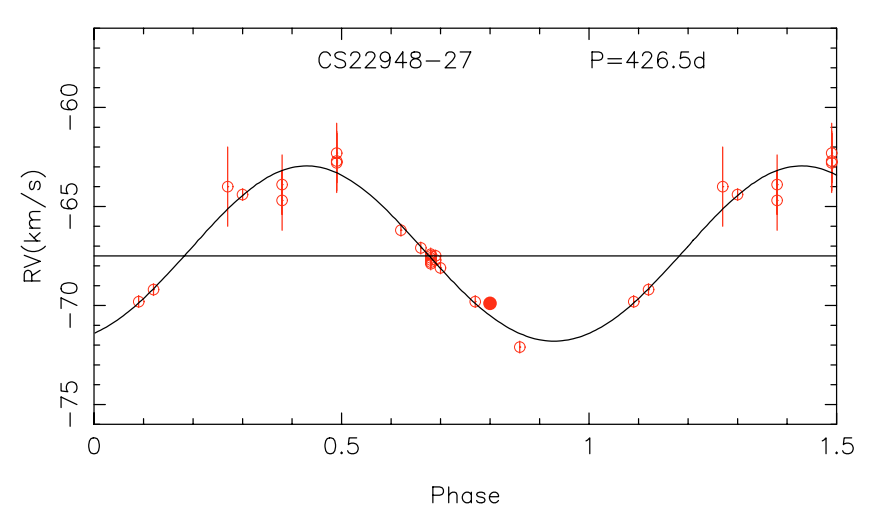

Fig. 1. Orbital variation of the radial velocity of CS 22948.27 computed from the orbital elements in Table 2 (the new observations are marked with filled circles). The period is close to $426.5 \mathrm{~d}$.

value. The orbital elements of CS 22948-27 (Table 2) were derived with the code "velocity" (Wichmann et al. 2003). The new period, achieving a satisfactory agreement of the individual measurements with the computed curve, has been found to be close to $426 \mathrm{~d}$. The new velocity curve generated with the orbital elements listed in Table 2 is displayed in Fig. 1.

\subsubsection{CS 29497-34}

In Fig. 2 we present the sequence of radial velocity measurements from 1995 to 2004 for CS 29497-34. From the last two values it appears now clearly that the radial velocity of CS 29497-34 is also variable. The orbital elements derived with the code "velocity" (Wichmann et al. 2003) are given in Table 2. The period has been found to be close to 4130 days. However the uncertainty is very large and more observations are needed to obtain a better precision in the determination of the orbital elements.

Finally, it is now established that the carbon-rich stars CS 22948-27 and CS 29497-34 are both binaries, both with long periods.

\section{Analysis}

\subsection{Model Atmospheres}

We used model atmospheres provided by the OSMARCS code, where LTE equilibrium, homogeneity and conservation of the total flux are assumed. It has been developed by

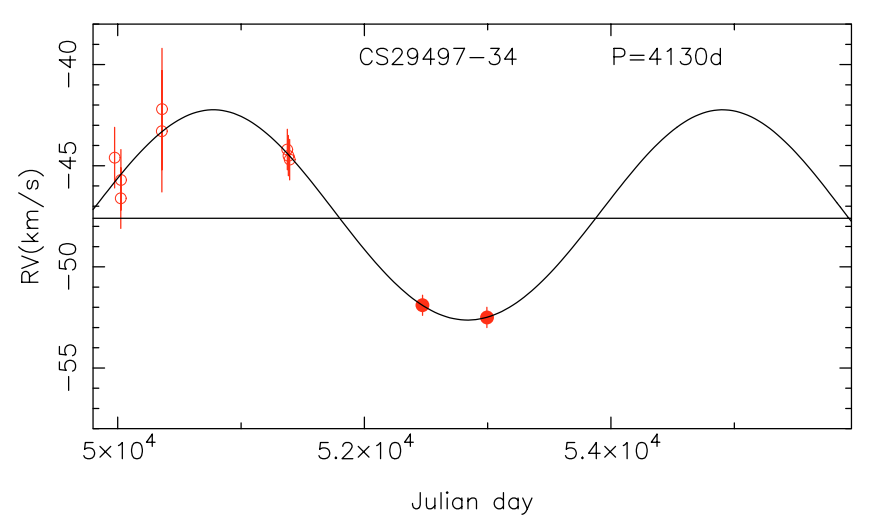

Fig. 2. Radial velocities of CS 29497-34 measured as a function of time. The abscissa is Julian date $-2400000 \mathrm{~d}$. The curve was generated by the orbital elements listed in Table 2 (the new observations are marked with filled circles). The period is close to $4130 \mathrm{~d}$.

Table 3. Stellar parameters effective temperatures, surface gravities, metallicities, and microturbulence velocities, as derived in Hill et al. (2000).

\begin{tabular}{cccccc}
\hline \hline & $\begin{array}{c}T_{\text {eff }} \\
(\mathrm{K})\end{array}$ & $\log g$ & {$\left[\mathrm{Fe}_{\mathrm{I}} / \mathrm{H}\right]$} & {$\left[\mathrm{Fe}_{\text {II }} / \mathrm{H}\right]$} & $\begin{array}{c}v_{\mathrm{t}} \\
\mathrm{km} \mathrm{s}^{-1}\end{array}$ \\
\hline CS 22948-27 & 4800 & 1.8 & -2.47 & -2.46 & 1.5 \\
$\mathrm{CS} 29497-34$ & 4800 & 1.8 & -2.90 & -2.91 & 1.5 \\
\hline
\end{tabular}

Gustafsson et al. (1975) and later improved by Plez et al. (1992), Edvardssson et al. (1993), Asplund et al. (1997) and Gustafsson et al. (2003). The models take into account the enhancement of the carbon and nitrogen abundances in the atmosphere. The atmospheric parameters (effective temperature $T_{\text {eff }}$, surface gravity $\log g$, metallicity $[\mathrm{Fe} / \mathrm{H}]$ and microturbulence velocity $v_{\mathrm{t}}$ ), adopted from Paper I, are given for convenience in Table 3.

\subsection{Carbon and nitrogen abundance}

A new determination of the abundance of carbon and nitrogen has been performed, using the $\mathrm{CH}$ lines based on line list by Luque \& Crosley (1999), and CN red lines as previously.

We assume abundances $\epsilon(\mathrm{C})=8.52$ and $\epsilon(\mathrm{N})=$ 7.92 (Grevesse \& Sauval 1998). The abundances obtained are $[\mathrm{C} / \mathrm{Fe}]=+2.43$ and $[\mathrm{N} / \mathrm{Fe}]=+1.75$ for CS $22948-27$ and $[\mathrm{C} / \mathrm{Fe}]=+2.63$ and $[\mathrm{N} / \mathrm{Fe}]=+2.38$ for $\mathrm{CS} 29497-34$. (Table 4).

\subsection{Abundances of metals from $\mathrm{Na}$ to $\mathrm{Zn}$}

In Barbuy et al. (1997) and Hill et al. (2000), due to crowding of lines, we could only determine the abundances of $\mathrm{C}$, $\mathrm{N}, \mathrm{Fe}, \mathrm{Na}$ and $\mathrm{Mg}$ and heavy elements. In the present paper the new higher resolution spectra of CS 22948-27 and CS 29497-34 allowed to measure equivalent widths of metallic lines from $\mathrm{Na}$ to $\mathrm{Zn}$. This abundance analysis was performed using Turbospectrum (Alvarez \& Plez 1998) and the results are given in Table 4. Solar abundances for the elemental species were adopted from Grevesse \& Sauval (1998). 
Table 4. Abundances of the elements in CS 22948-27 and CS 29497-34.

\begin{tabular}{|c|c|c|c|c|c|}
\hline Elem. & $\log \epsilon_{*}$ & {$[\mathrm{M} / \mathrm{H}]$} & {$[\mathrm{M} / \mathrm{Fe}]$} & $\sigma$ & $N$ \\
\hline \multicolumn{6}{|c|}{ CS 22948-27 $[\mathrm{Fe} / \mathrm{H}]=-2.47$} \\
\hline C & 8.49 & -0.03 & +2.43 & & Synt \\
\hline $\mathrm{N}$ & 7.21 & -0.71 & +1.75 & & Synt \\
\hline $\mathrm{Na} \mathrm{I}^{*}$ & 4.43 & -1.90 & +0.57 & 0.17 & 2 \\
\hline $\mathrm{Mg} \mathrm{I}$ & 5.42 & -2.16 & +0.31 & 0.28 & 4 \\
\hline $\mathrm{Al} \mathrm{I} *$ & 3.85 & -2.62 & -0.15 & & 1 \\
\hline $\mathrm{Ca} \mathrm{I}$ & 4.43 & -1.93 & +0.54 & 0.15 & 9 \\
\hline $\mathrm{Ti} \mathrm{I}$ & 2.89 & -2.13 & +0.34 & 0.28 & 10 \\
\hline TiII & 3.05 & -1.97 & +0.50 & 0.20 & 20 \\
\hline $\mathrm{Cr} \mathrm{I}$ & 3.05 & -2.62 & -0.15 & 0.10 & 3 \\
\hline Ni I & 3.77 & -2.48 & -0.01 & & 1 \\
\hline $\mathrm{Zn} \mathrm{I}$ & 2.49 & -2.11 & +0.36 & & 1 \\
\hline Sr II & 1.35 & -1.57 & +0.90 & & Synt \\
\hline Y II & 0.87 & -1.47 & +1.00 & & Synt \\
\hline Ba II & 1.92 & -0.21 & +2.26 & & Synt \\
\hline La II & 1.02 & -0.15 & +2.32 & & Synt \\
\hline Ce II & 1.31 & -0.27 & +2.20 & & Synt \\
\hline Pr II & 0.08 & -0.63 & +1.87 & & Synt \\
\hline Nd II & 1.25 & -0.25 & +2.22 & & Synt \\
\hline Eu II & -0.08 & -0.59 & +1.88 & & Synt \\
\hline Dy II & 0.27 & -0.87 & +1.60 & & Synt \\
\hline $\mathrm{Pb} \mathrm{I}$ & 2.20 & +0.25 & +2.72 & & Synt \\
\hline \multicolumn{6}{|c|}{ CS 29497-34 [Fe/H] = -2.90 } \\
\hline $\mathrm{C}$ & 8.24 & -0.28 & +2.63 & & Synt \\
\hline $\mathrm{N}$ & 7.39 & -0.53 & +2.38 & & Synt \\
\hline $\mathrm{Na} \mathrm{I*}$ & 4.61 & -1.72 & +1.18 & 0.19 & 2 \\
\hline $\mathrm{Mg} \mathrm{I}$ & 5.40 & -2.18 & +0.72 & 0.34 & 4 \\
\hline $\mathrm{Al} \mathrm{I}^{*}$ & 3.56 & -2.91 & -0.01 & & 1 \\
\hline $\mathrm{Ca} \mathrm{I}$ & 3.91 & -2.45 & +0.45 & 0.18 & 9 \\
\hline Ti I & 2.41 & -2.61 & +0.29 & 0.28 & 11 \\
\hline TiII & 2.56 & -2.46 & +0.44 & 0.27 & 15 \\
\hline $\mathrm{Cr} I$ & 2.54 & -3.13 & -0.23 & 0.12 & 3 \\
\hline Ni I & 3.36 & -2.89 & +0.01 & & 1 \\
\hline $\mathrm{Zn} \mathrm{I}$ & 2.15 & -2.44 & +0.46 & & 1 \\
\hline Sr II & 1.02 & -1.90 & +1.00 & & Synt \\
\hline Y II & 0.54 & -1.80 & +1.10 & & Synt \\
\hline Ba II & 1.26 & -0.87 & +2.03 & & Synt \\
\hline La II & 0.39 & -0.78 & +2.12 & & Synt \\
\hline $\mathrm{Ce}$ II & 0.63 & -0.95 & +1.95 & & Synt \\
\hline Pr II & -0.37 & -1.08 & +1.82 & & Synt \\
\hline Nd II & 0.69 & -0.81 & +2.09 & & Synt \\
\hline $\mathrm{Eu}$ II & -0.59 & -1.10 & +1.80 & & Synt \\
\hline Dy II & -0.26 & -1.40 & +1.50 & & Synt \\
\hline $\mathrm{Pb} \mathrm{I}$ & 2.00 & +0.05 & +2.95 & & Synt \\
\hline
\end{tabular}

* To take into account the NLTE effects the abundances of Na given in the table have to be decreased by 0.5 dex and the abundances of $\mathrm{Al}$ have to be increased by 0.65 dex.
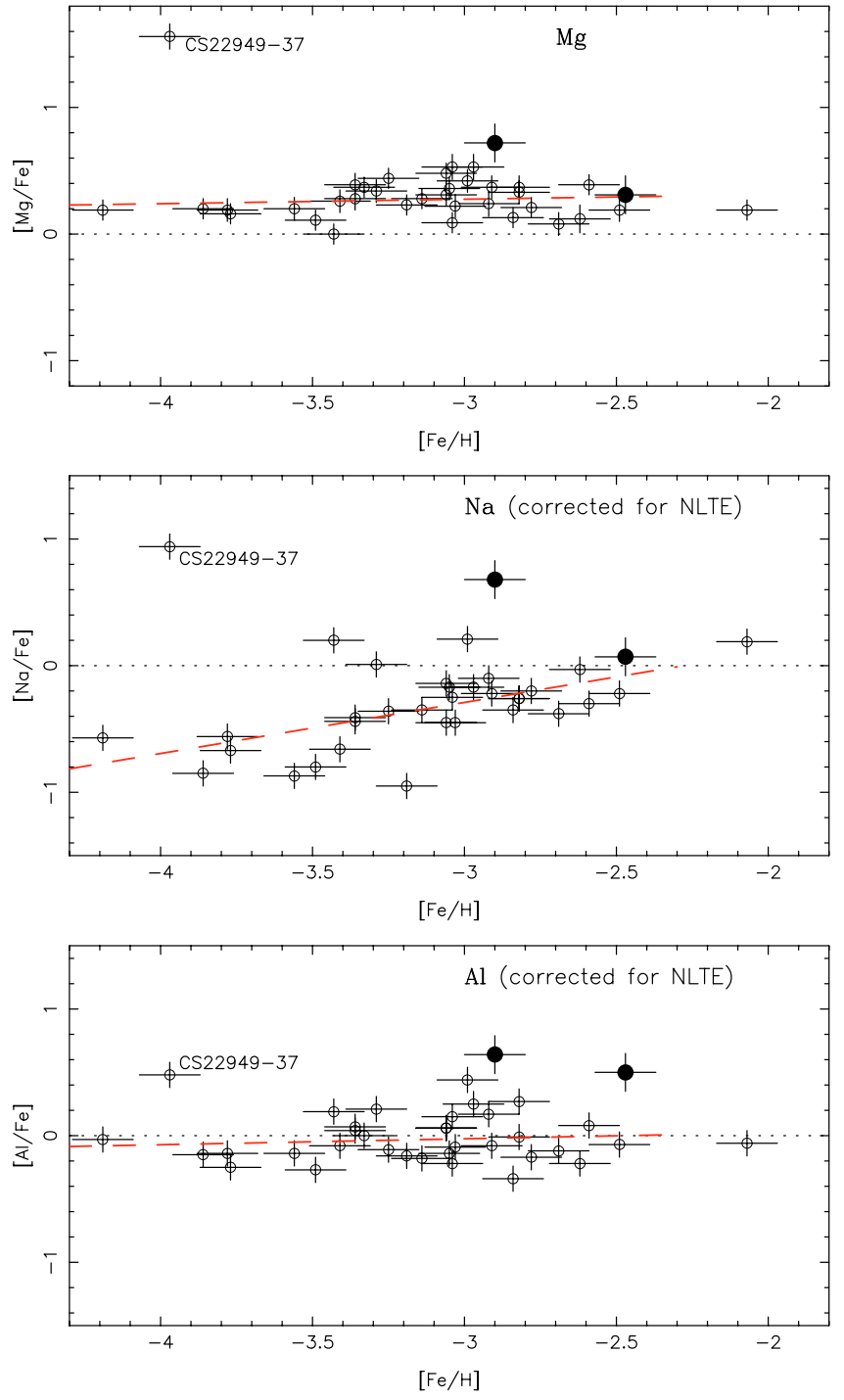

Fig. 3. Abundances of $\mathrm{Mg}, \mathrm{Na}$ and $\mathrm{Al}$ in $\mathrm{CS} 22948-27([\mathrm{Fe} / \mathrm{H}]=$ -2.47) and CS 29497-34 ([Fe/H] = -2.90) (filled circles) compared to the abundances of these elements measured in extremely metal-poor stars (open circles). CS 22949-37 (Depagne et al. 2002) is also a carbon rich star but its very peculiar pattern is explained by the ejecta of a zero-metal SNII with large fallback.

The sodium and the aluminum abundances are computed from the resonance doublets: Na D lines at 5890 and $5896 \AA$ and the $\mathrm{Al}$ line at $3961.5 \AA$ (the other line of the aluminum doublet is too severely blended to be used). All these lines are sensitive to NLTE effects (Cayrel et al. 2004; Baumüller et al. 1998; Baumüller \& Gehren 1997; Norris et al. 2001). As a consequence the abundance of sodium given in Table 4 should be decreased by $0.5 \mathrm{dex}$ and that of aluminum should be increased by about 0.65 dex (in Fig. 3 the corrected values are used).

We have compared the abundances of metals in CS 22948-27 and CS 29497-34 to the abundances found in Cayrel et al. (2004) for extremely metal poor stars (Fig. 3). There is rather good agreement for $\mathrm{Ca}, \mathrm{Ti}, \mathrm{Cr}, \mathrm{Ni}$ and $\mathrm{Zn}$, whereas CS 29497-34 is Mg-rich, Na-rich and Al-rich. In CS 22948-27 the abundance of $\mathrm{Mg}$ and $\mathrm{Na}$ are pratically as expected for a normal star with $[\mathrm{Fe} / \mathrm{H}]=-2.5$ (i.e. showing 
the classical enhancement of the $\alpha$-elements), whereas the abundance of $\mathrm{Al}$ is enhanced.

Figure 3 shows $[\mathrm{Mg} / \mathrm{Fe}], \mathrm{Na} / \mathrm{Fe}]$ and $[\mathrm{Al} / \mathrm{Fe}]$ vs. $[\mathrm{Fe} / \mathrm{H}]$ in CS 22948-27 and CS 29497-34, compared with the abundances of these elements measured in extremely metal-poor stars. In this figure, CS 22949-37 (Depagne et al. 2002) is also plotted illustrating its peculiar characteristics: it is carbon rich, and its pattern can be explained as typical ejecta of a massive zero-metal SNII with large fallback, according to Woosley \& Weaver (1995), and a $Z=10^{-4}$ according to Umeda \& Nomoto (2003).

\subsection{Heavy element abundances}

The heavy element abundances have been determined by spectrum synthesis calculations. The codes Turbospectrum described by Alvarez \& Plez (1998), and that described by Barbuy et al. (2003) were both used.

Absorption lines of the following molecules were taken into account in the calculations: $\mathrm{MgH}\left(\mathrm{A}^{2} \Pi-\mathrm{X}^{2} \Sigma\right), \mathrm{C}_{2}\left(\mathrm{~A}^{3} \Pi-\mathrm{X}^{3} \Pi\right)$, $\mathrm{CN}$ blue $\left(\mathrm{B}^{2} \Sigma-\mathrm{X}^{2} \Sigma\right), \mathrm{CH}\left(\mathrm{A}^{2} \Delta-\mathrm{X}^{2} \Pi\right), \mathrm{CH}\left(\mathrm{B}^{2} \Delta-\mathrm{X}^{2} \Pi\right)$, and $\mathrm{CN}$ red $\left(\mathrm{A}^{2} \Pi-\mathrm{X}^{2} \Sigma\right)$.

For lines of the heavy elements BaII, LaII and EuII, a hyperfine structure was taken into account, based on the hyperfine constants by Lawler et al. (2001a) for EuII, Lawler et al. (2001b) for LaII and Biehl (1976) and McWilliam (1998) for BaII. For the $\mathrm{PbI} 4057.807$ line, the hyperfine structure given in Van Eck et al. (2003) was adopted.

For most of the heavy elements our new determinations (Table 4) confirm the values found in Paper I. For the determination of the Pr abundance we could add the blue lines at $3964.3,3964.8$ and $3965.3 \AA$ and we found an abundance slightly higher than that of Paper I. Moreover we revised the abundance of europium and we could determine, for the first time, the abundance of $\mathrm{Pb}$ in these stars.

\subsubsection{Eu}

The abundance of Eu in these cool carbon-rich stars is best determined from the red lines at 6437.64 and $6645.13 \AA$ but an unidentified line appears more clearly in the present data on the blue side of the $6645.13 \AA$ line, with the result that, relative to Paper $\mathrm{I},[\mathrm{Eu} / \mathrm{Fe}]$ is decreased from +2.10 to +1.88 in CS 22948-27 and from +2.25 to +1.8 in CS 29497-34 (Fig. 4).

We tried to use the blue europium lines at 4129 and $4205 \AA$ to estimate its isotopic ratio. In s-process element-enhanced metal-poor stars, this ratio has been always found to be close to the solar value (see Sneden et al. 2002; Aoki et al. 2003, hereafter AR03), but it would be interesting to check this ratio in $\mathrm{r}$ and s-process enhanced stars like CS 22948-27 and CS 29497-34. However the blue EuII lines in these stars are severely blended with strong $\mathrm{CN}$ features, and do not allow the derivation of isotopic ratios.

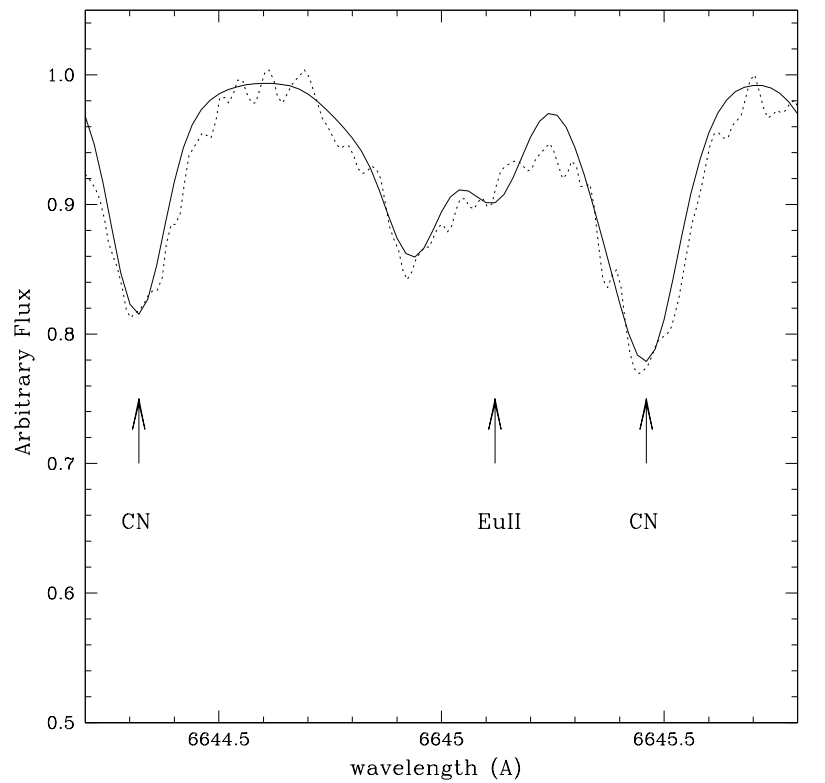

Fig. 4. CS 29497-34: EuII 6645 line computed with $\log \epsilon(\mathrm{Eu})=-0.60$ $([\mathrm{Eu} / \mathrm{Fe}]=+1.8)$.
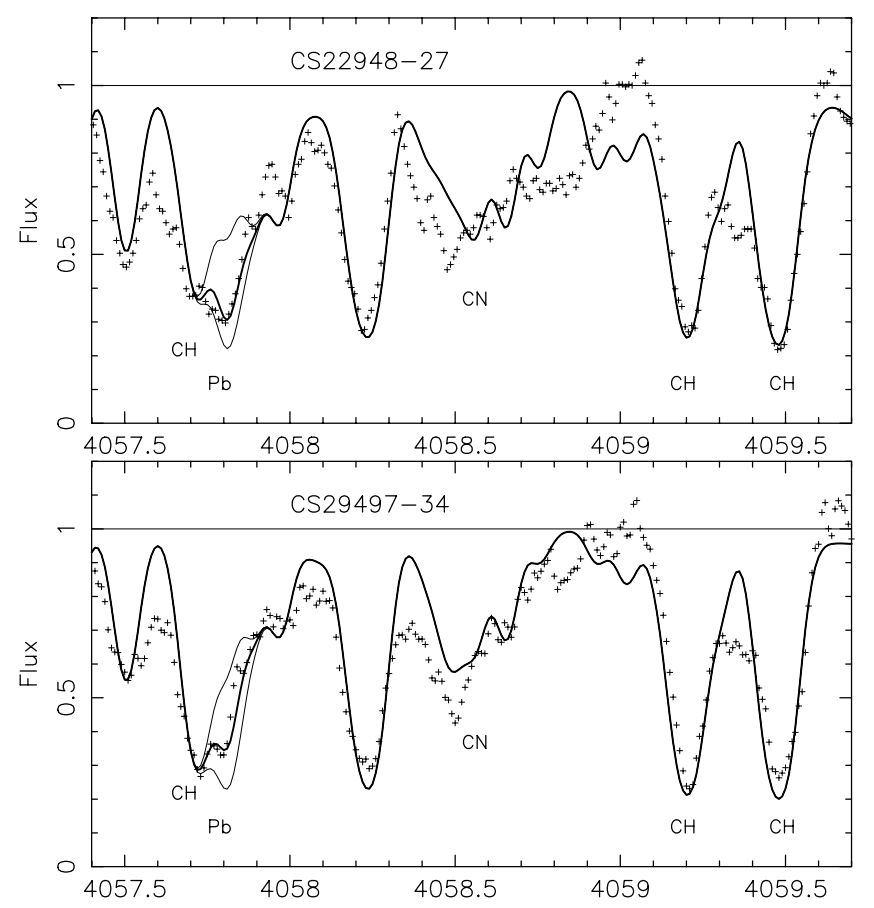

Fig. 5. $\mathrm{Pb}$ I line computed with $\log \epsilon(\mathrm{Pb})=1.5,2.5$ and the adopted value $\left(\log \epsilon(\mathrm{Pb})=2.2\right.$ for CS 22948-27, and $\log \epsilon_{\mathrm{Pb}}=2.0$ for CS 29497-34. These values correspond to $[\mathrm{Pb} / \mathrm{Fe}]_{22948-27}=+2.7$ and $[\mathrm{Pb} / \mathrm{Fe}]_{29497-34}=+2.95$.

\subsection{2. $\mathrm{Pb}$}

We were able to determine the abundance of $\mathrm{Pb}$, thanks to the high resolving power of the present observations. Figure 5 shows the fit to the PbI 4057.8 $\AA$ line in both stars. The overabundance is very large: the best fit in CS 22948-27 is obtained for $\log \epsilon(P b)=+2.2([\mathrm{~Pb} / \mathrm{Fe}]=+2.72)$ and in CS 29497-34 for $\log \epsilon(P b)=+2.00([\mathrm{~Pb} / \mathrm{Fe}]=+2.95)$. 


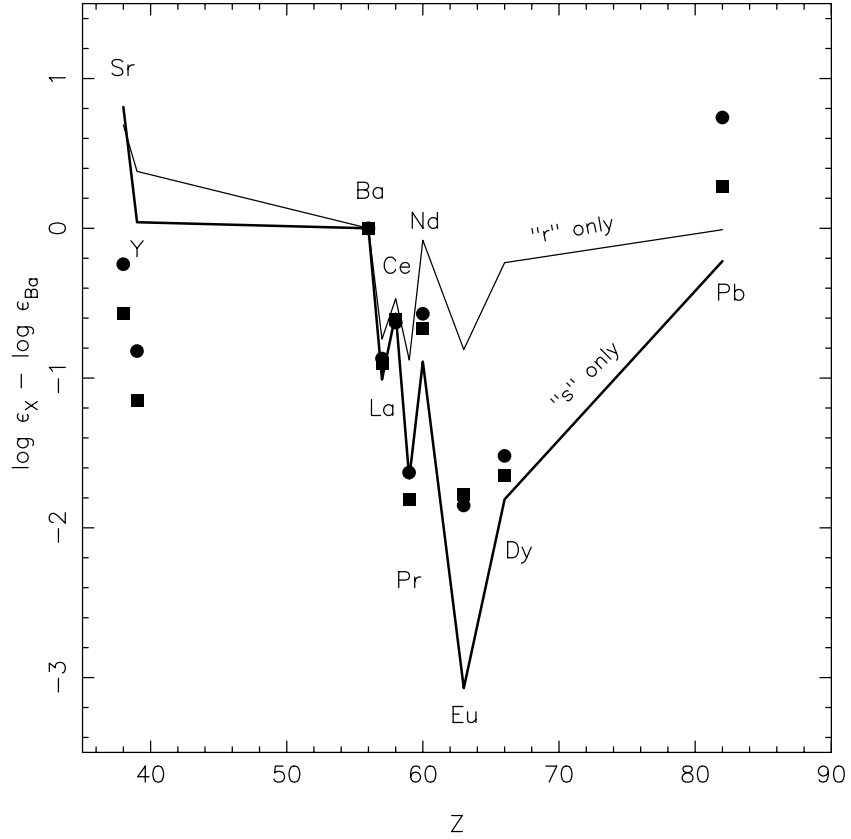

Fig. 6. Abundances relative to $\mathrm{Ba}$ [X/Ba] in CS 22948-27 (filled square) and CS 29497-34 (filled circle) is compared to the r- and s-process solar abundances (thin and thick lines) from Burris et al. (2000). Note that in this figure we have plotted only the pattern of the elements really measured in the stars.

\subsubsection{General pattern of the heavy elements}

In Fig. 6 the patterns of the heavy elements relative to $\mathrm{Ba}$ in CS 22948-27 and CS 29497-34 are compared to the r- and s-process solar abundances from Burris et al. (2000). The abundances of the lighter elements Sr and Y seem to be discrepant: the enhancements in $\mathrm{Sr}$ and $\mathrm{Y}$ are much lower than the enhancements in $\mathrm{Ba}$ (Table 4) and consequently the ratios $\mathrm{Y} / \mathrm{Ba}$ and $\mathrm{Sr} / \mathrm{Ba}$ appear particularly low. On the contrary, the enhancement of $\mathrm{Pb}$ is larger than the enhancement of $\mathrm{Ba}$. For the elements heavier than $\mathrm{Ba}$ (excluding $\mathrm{Pb}$ ), the pattern of the elements is close to the solar "s only curve", but generally a little higher. Europium in particular is very rich in CS 22948-27 and CS 29497-34, indicating that the observed pattern implies a contribution from both s- and r-processes. The numerical values of the observed ratios $[\mathrm{Ba} / \mathrm{Eu}]$ (see Table 5), intermediate between the values for r-only- and s-only process in the solar system, lead to the same conclusion. Owing to the deviations of $\mathrm{Sr}, \mathrm{Y}$ and $\mathrm{Pb}$, the general pattern is clearly non-solar. These peculiarities are also observed in similar stars (AR02; CC03). One of the causes of the non-solar pattern is the metal deficiency of these stars, implying a higher ratio of neutrons relative to seed nuclei (higher neutron flux). Quantitatively, an indicator of the neutron flux [hs/ls] (see Table 5) is defined as the ratio of the heavy- to the light-"s" elements, relative to the solar ratio. It is computed as in Norris et al. (1997) as $\langle[\mathrm{Ba} / \mathrm{Fe}]$, $[\mathrm{Ce} / \mathrm{Fe}],[\mathrm{Nd} / \mathrm{Fe}]\rangle /\langle[\mathrm{Sr} / \mathrm{Fe}],[\mathrm{Y} / \mathrm{Fe}],[\mathrm{Zr} / \mathrm{Fe}]\rangle$; note that $\mathrm{Zr}$ has been omitted in our calculation of this quantity. The theoretical models predict an increase of the [hs/ls] ratio with decreasing metallicity $[\mathrm{Fe} / \mathrm{H}]$, and Busso et al. (2001) discuss the limitations of this correlation.

\section{Discussion of the abundances}

It is interesting to compare our stars to other ones found in the literature for being rich in both $\mathrm{s}-(\mathrm{Pb}$-rich) and r-process elements, in particular HE 2148-1247 analyzed by CC03, and the stars homogeneously analyzed by Aoki et al. (2001, 2002a,b, hereafter AR01 and AR02). From the sample of metal-poor, carbon-rich and Pb-rich stars of AR01 and AR02, we have disconsidered two stars: one because of its relatively high metallicity (we kept the stars with $[\mathrm{Fe} / \mathrm{H}]<-2.2$ ) and the other because only an upper limit of the $\mathrm{Pb}$ abundance had been determined. All these stars are both s-rich and r-rich: we will designate these stars by " $\mathrm{r}+\mathrm{s}$ stars". The first 4 stars of AR02 in Table 5 are the most r-rich, according to their $[\mathrm{Ba} / \mathrm{Eu}]$ ratio (see however AR03), two of them have variable radial velocities.

In Table 5 the abundances of $\mathrm{Ba}, \mathrm{La}, \mathrm{Ce}, \mathrm{Eu}$ and $\mathrm{Pb}$ in CS 22948-27 and CS 29497-34 are listed, along with those of HE 2148-1247 (CC03) and the AR01 and AR02 ones. One of the common points of the stars in Table 5, enhanced in $\mathrm{r}$ - and s-process elements, is a large abundance of $\mathrm{Pb}$. The metal-poor $\mathrm{Pb}$-rich stars are not many: a recent review is given in Sivarani et al. (2004), where 25 such stars are reported to be known (but not all of them are "r-rich"). In Table 5 we also added, for comparison purposes, a very metal-poor star dominated by the s-process and not r-rich: HE 024-2523 (Lucatello et al. 2003).

The two stars analyzed here are very similar to HE 21481247 (CC03). These stars are long period binaries (and presumably also the third one), they are metal-poor, enhanced in carbon, nitrogen, $\alpha$ elements, r-process and s-process elements (especially in $\mathrm{Pb}$ ). They have measured abundances for a number of elements and form a homogeneous group

In Fig. 7 the abundance patterns of CS 22948-27 and CS 29497-34 are compared to those of HE 148-1247 (CC03) and one star from AR02: CS 22898-27 (the eight AR01, AR02 stars in Table 5 are essentially similar and we chose to plot only one of them for the lisibility of the figure).

In these stars the s-process elements have probably been transferred from an AGB companion. In very metal-poor AGBs, the s-process operates with a high ratio of neutrons to seed nuclei that should favor a high abundance of $\mathrm{Pb}$. In fact, according to Gallino et al. (1998), a large production of the double magic nucleus ${ }^{208} \mathrm{~Pb}$ is due to AGB stars of low metallicity, which explains the presence of $\mathrm{Pb}$ in low-metallicity stars, as well as the fraction of this isotope in the Sun. This is confirmed by Travaglio et al. (2001), together with the fact that, on the other hand, low mass AGB stars $\left(M \leq 4 M_{\odot}\right)$ play the dominant role in the production of s-elements in the Galaxy. Calculations made by Goriely \& Mowlawi (2000) and Goriely $\&$ Siess (2001) predict as well large enhancements of third peak s-elements relative to first and second peaks. The enhancement of $\mathrm{Pb}$ in metal-poor stars has been later confirmed by observations (Van Eck 2001).

\subsection{Neutron flux and $\mathrm{Pb}$ abundance}

Figure 8 compares the observed $\mathrm{Pb} / \mathrm{Ce}$ ratios of our stars, together with the other "r-s-rich" stars in Table 5, with model predictions of the ejecta of metal-poor AGBs (Van Eck et al. 2003; 
Table 5. Metallicities and abundances of $\mathrm{Ba}, \mathrm{Pb}$ and $\mathrm{Eu}$, and the $[\mathrm{hs} / \mathrm{ls}]$ measure of second peak to first peak s-elements, in selected very metal-poor stars $([\mathrm{Fe} / \mathrm{H}]<-2.2)$, showing enhancements of both s- and r-process elements, and carbon-rich. HE 0024-2523 is a metal-poor s-rich star given here for comparison. The ratios take into account a solar Fe abundance of 7.50, and a renormalization of results in the literature was applied. The references are given in the last column.

\begin{tabular}{lccllllll}
\hline \hline Star & {$[\mathrm{Fe} / \mathrm{H}]$} & {$[\mathrm{Ba} / \mathrm{Fe}]$} & {$[\mathrm{La} / \mathrm{Fe}]$} & {$[\mathrm{Ce} / \mathrm{Fe}]$} & {$[\mathrm{Eu} / \mathrm{Fe}]$} & {$[\mathrm{Pb} / \mathrm{Fe}]$} & {$[\mathrm{hs} / \mathrm{ls}]$} & $\mathrm{Ref}$. \\
\hline "s-" and "r-rich" stars & & & & & & & \\
CS 22948-27 & -2.47 & 2.26 & 2.32 & 2.20 & 1.88 & 2.72 & 0.97 & 1,2 \\
CS 29497-34 & -2.90 & 2.03 & 2.12 & 1.95 & 1.80 & 2.95 & 1.28 & 1,2 \\
& & & & & & & & \\
HE 2148-1247 & -2.30 & 2.36 & 2.38 & 2.28 & 1.98 & 3.12 & 1.76 & 3 \\
& & & & & & & & \\
CS 29526-110 & -2.38 & 2.20 & 1.74 & 2.06 & 1.77 & 3.43 & - & 4 \\
CS 22898-027 & -2.25 & 2.33 & 2.19 & 2.19 & 1.93 & 2.96 & 1.37 & 4 \\
CS 31062-012 & -2.55 & 2.07 & 2.07 & 2.17 & 1.66 & 2.50 & 1.51 & 4 \\
CS 31062-50 & -2.32 & 2.40 & 2.49 & 2.15 & 1.88 & 2.97 & - & 4 \\
HD 196944 & -2.25 & 1.20 & 0.97 & 1.07 & 0.22 & 2.01 & 0.29 & 4 \\
CS 30301-015 & -2.64 & 1.53 & 0.88 & 1.20 & 0.22 & 1.78 & 0.99 & 4 \\
LP 625-44 & -2.71 & 2.84 & 2.52 & 2.33 & 2.00 & 2.66 & 1.01 & 5 \\
LP 625-44 & -2.72 & 2.81 & 2.40 & 2.22 & 1.72 & 2.60 & 1.30 & 6 \\
LP 706-7 & -2.74 & 2.11 & 1.87 & 1.92 & 1.45 & 2.40 & 1.76 & 5 \\
\hline "s-rich" star & & & & & & & & \\
HE 0024-2523 & -2.70 & 1.46 & 1.80 & - & $<1.10$ & 3.30 & - & 7 \\
\hline
\end{tabular}

References: 1 present work; 2 Paper I; 3 CC03; 4 Aoki et al. (2002a); 5 AR01; 6 AR02 7 Lucatello et al. (2003).

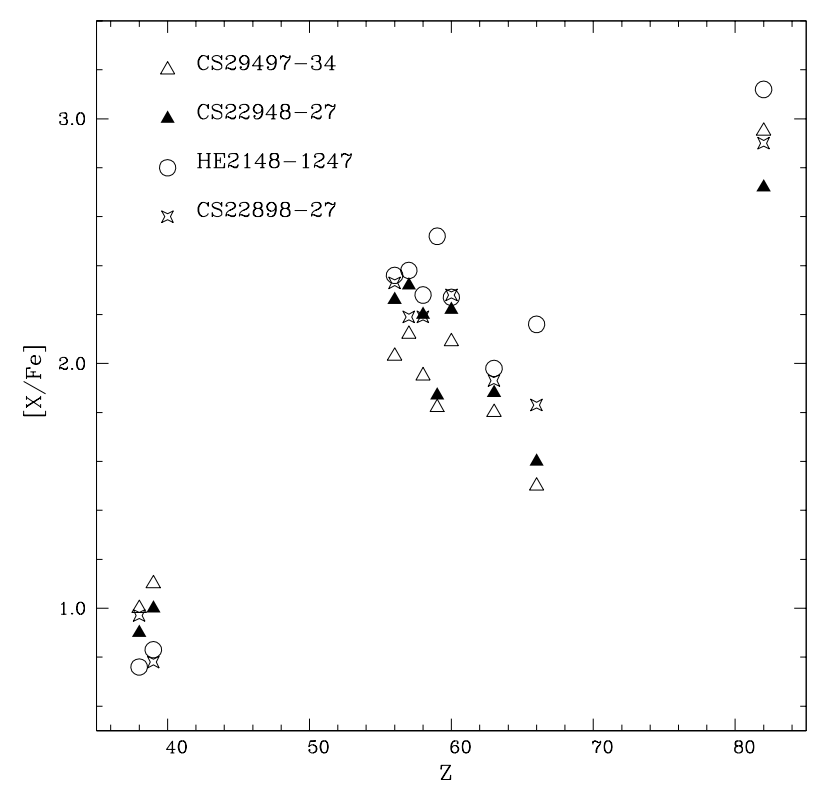

Fig. 7. Abundances of the heavy elements in CS 22948-27 (filled triangles) and CS 29497-34 (open triangles), compared to HE 148-1247 (open circles) and one of the AR01, AR02 stars CS 22898-27 (open stars). All the stars here have a very similar pattern.

Goriely \& Mowlavi 2000; Goriely \& Siess 2001). These models predict too high values of $[\mathrm{Pb} / \mathrm{Ce}]$ and $[\mathrm{Pb} / \mathrm{La}]$ (see Fig. 8).
The same conclusion was reached by Van Eck et al. (2003) for classical "s-rich" metal-poor stars.

In Fig. 9, [hs/ls] is plotted vs. [Fe/H] for the $\mathrm{Pb}$ and r-rich stars reported in Table 5. No clear correlation is observed. For most of the stars [hs/ls] seems to increase with decreasing metallicity but there are exceptions: HD196944 has a very low [hs/ls] for its metallicity and on the contrary [hs/ls] is very high in LP 706-7.

Figures 10 and 11 show $[\mathrm{Pb} / \mathrm{Ba}]$ vs. $[\mathrm{Fe} / \mathrm{H}]$ and $[\mathrm{Pb} / \mathrm{Eu}]$ vs. $[\mathrm{Fe} / \mathrm{H}]$ respectively, for the sample of Table 5 . Whereas there might be a correlation of lead-to-barium (representing a third peak to a second peak s element ratio) with metallicity, the same is not true for the lead-to-europium (a third peak selement relative to an r-element) ratio. The absence (or the low degree) of correlation indicates that the amount of $\mathrm{Pb}$ produced varies from one site to another, and from star-to-star, as noted by CC03 and explained by models (e.g. Busso et al. 1999).

A close look at the ratios $[\mathrm{Pb} / \mathrm{Ce}]$ versus [hs/ls] shows the lack of a clear correlation: for example, compared to LP706-7, the other star (LP 625-44) of AR01 has a $\mathrm{Pb} / \mathrm{Ce}$ ratio larger by a factor ten, but a ratio hs/ls smaller by a factor 5 , showing for these two stars an anticorrelation. The neutron flux, derived from the comparison of elements of the first and second peak, has no clear correlation with the abundance of $\mathrm{Pb}$ (a third-peak element). More generally, no clear correlations were found between the $\mathrm{Pb}$ abundance and other basic parameters, owing to the sensitivity of this abundance to a number of critical 

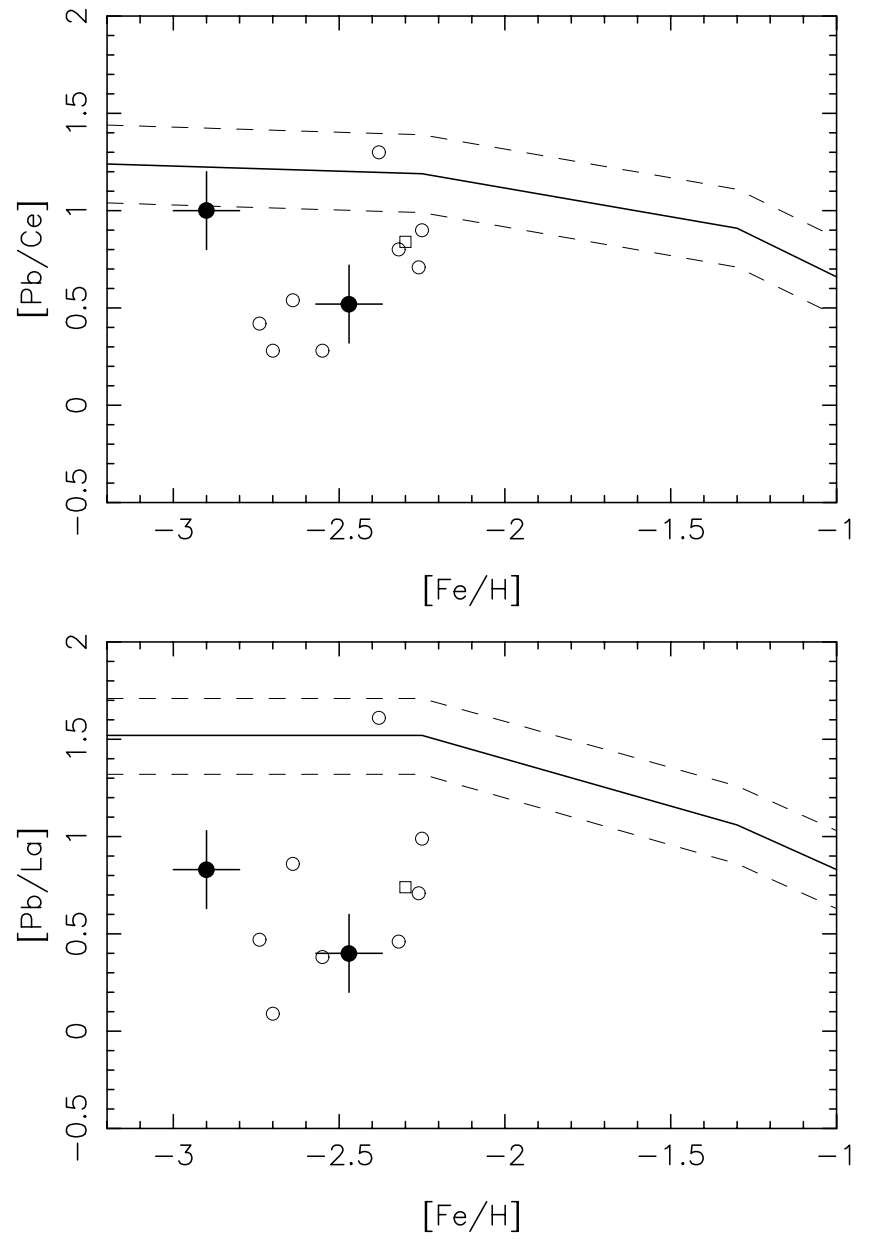

Fig. 8. $[\mathrm{Pb} / \mathrm{Ce}]$ and $[\mathrm{Pb} / \mathrm{La}]$ versus $[\mathrm{Fe} / \mathrm{H}]$ for $\mathrm{CS} 22948-27$ and CS 29497-34 (filled circles), HE 148-1247 (open square), and the AR01, AR02, AR03 (open circles) selected in Table 5. Predictions from the standard PMP model from Goriely \& Mowlavi (2000) and Goriely \& Siess (2001) are represented by the solid line. The dashed lines provide a rough estimate of the uncertainties of these predictions (see Van Eck et al. 2003). The model predicts generally a too high ratio of $[\mathrm{Pb} / \mathrm{Ce}]$ and $[\mathrm{Pb} / \mathrm{La}]$.

parameters (for example the concentration and the profile of the ${ }^{13} \mathrm{C}$ pocket, e.g. Busso et al. 1999).

\subsection{The heavy elements in metal-poor stars}

In normal stars, the evolution of heavy element abundances vs. iron (Burris et al. 2000) shows that the onset of the r-process (attributed to low mass SN II) is observed at about $[\mathrm{Fe} / \mathrm{H}]=$ -2.9 and that of the s-process (attributed to massive AGBs) at $[\mathrm{Fe} / \mathrm{H}]=-2.75$, near the metallicities of the two stars analysed here (and the one by $\mathrm{CC} 03$ ). The ratios $\mathrm{X} / \mathrm{Fe}$ (where $\mathrm{X}$ means any heavy element) are widely scattered at low metallicities. The large scatter observed for the abundances of the s-elements at low metallicity (especially for $\mathrm{Sr}$ ) are the signature of strong inhomogeneities in the interstellar matter which formed the analyzed stars. Some metal-poor stars are found with somewhat enhanced "s" or " $r$ " elements, indicating a nucleosynthesis pattern formed just after the onset of the " $\mathrm{r}$ " and " $\mathrm{s}$ " processes.

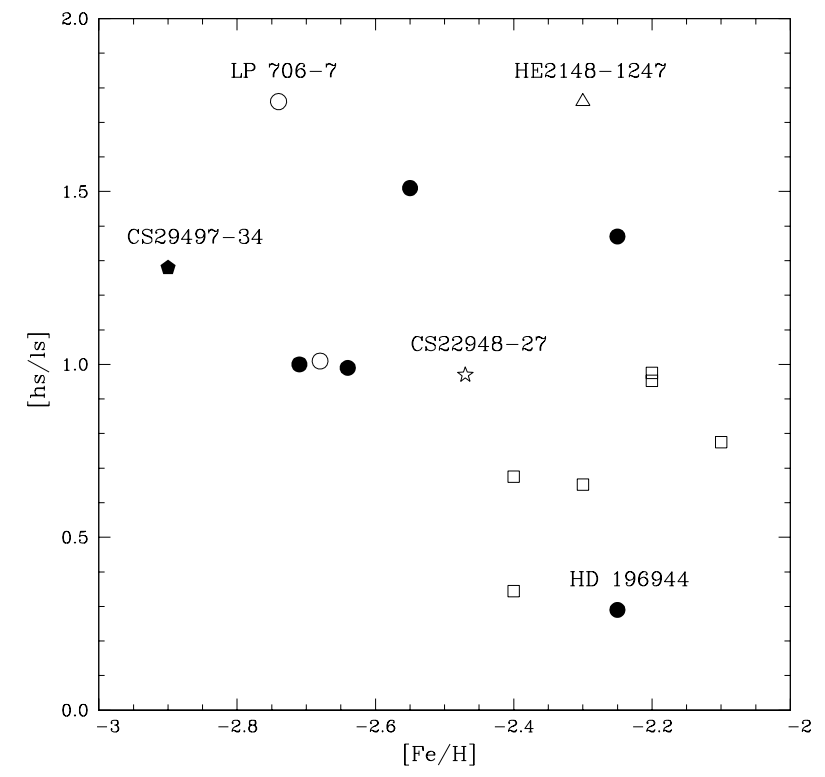

Fig. 9. $[\mathrm{hs} / \mathrm{ls}]$ vs. $[\mathrm{Fe} / \mathrm{H}]$ for $\mathrm{Pb}-$ rich stars. Symbols: filled pentagone:CS 29497-34, open star: CS 22948-27, open triangle HE 2148-1247 (CC03), open circles: AR01, filled circles: AR02, open squares: Van Eck et al. (2003).

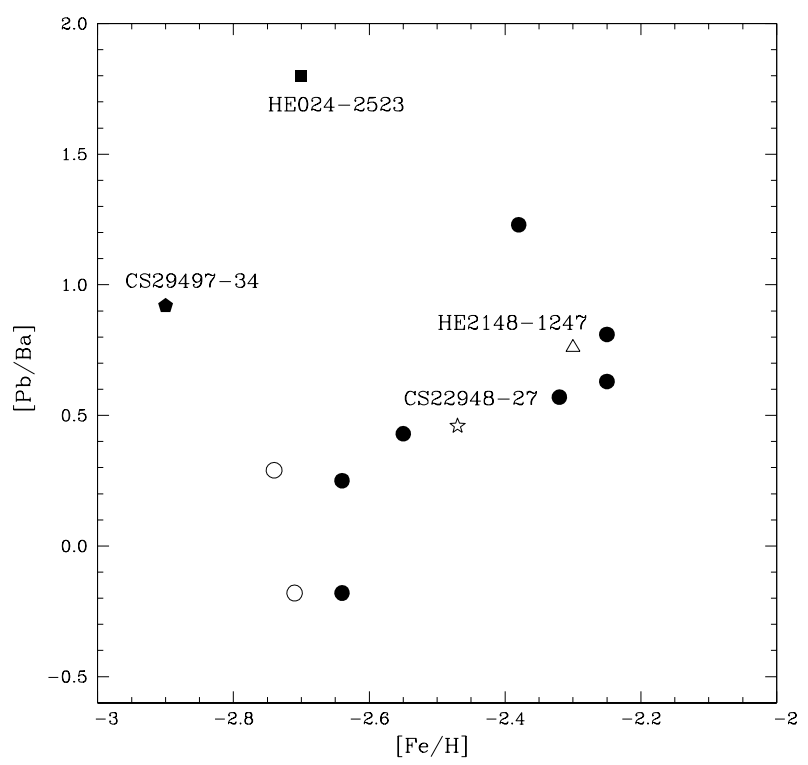

Fig. 10. $[\mathrm{Pb} / \mathrm{Ba}]$ vs. $[\mathrm{Fe} / \mathrm{H}]$ for Pb-rich stars. Symbols: filled square: HE 024-2523 (Lucatello et al. 2003), and others are the same as in Fig. 9.

The $\mathrm{r}+\mathrm{s}$ rich stars, in contrast, show very large enhancements, that have to be explained.

\subsection{The r-process elements in metal-poor stars}

In a few stars, it happens that the heavy elements are due to the r-process only (Burris et al. 2000), very few of these stars are extremely metal-poor: the most extreme examples are CS 22892-052 (Sneden et al. 1996, 2003), and CS 31082-001 (Cayrel et al. 2001; Hill et al. 2002). They have $[\mathrm{Eu} / \mathrm{Fe}]=1.5$ and 1.7 respectively, larger than any normal metal-poor stars 


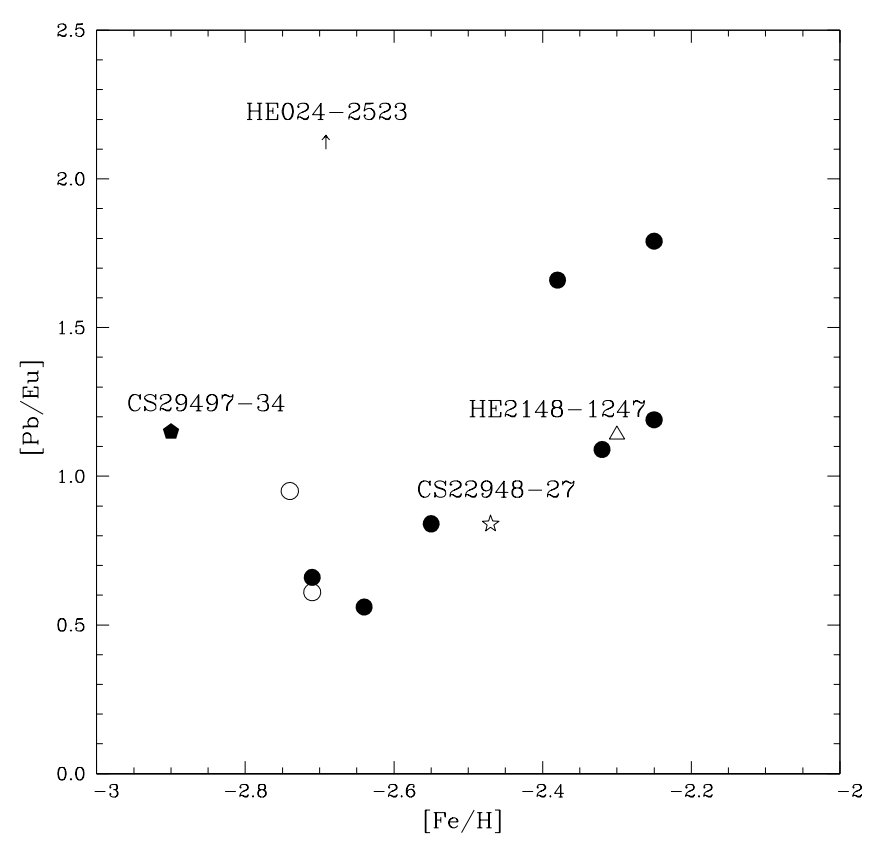

Fig. 11. $[\mathrm{Pb} / \mathrm{Eu}]$ vs. $[\mathrm{Fe} / \mathrm{H}]$ for $\mathrm{Pb}$-rich stars. Symbols: same as in Fig. 9.

(Honda et al. 2004). CS 22892-052 is also carbon-rich. By comparison, the two stars analyzed here, together with the one analyzed by $\mathrm{CCO} 3$, have even larger ratios: $[\mathrm{Eu} / \mathrm{Fe}]=1.88$, 1.80 and 1.98 dex respectively. Some other C-rich $\mathrm{r}+\mathrm{s}$ stars observed by AR01, AR02 have also very large [Eu/Fe] ratios: e.g. LP 625-44 reaches 2.00 dex.

The large $[\mathrm{Eu} / \mathrm{Fe}]$ ratios observed in the $\mathrm{r}+\mathrm{s}$ stars could be understood by the addition, to a already r-rich matter, of a large amount of s-rich matter: such matter includes of course a lot of $\mathrm{Ba}$, but also some $\mathrm{Eu}$, and a simple calculation shows that, if the exceptional stars strongly enhanced in r-process elements only receive large amounts of s-process elements they will reach $[\mathrm{Eu} / \mathrm{Fe}]$ ratios similar to those of the $\mathrm{r}+\mathrm{s}$ stars. AR02 considered that this process of addition could possibly explain the formation of the $\mathrm{r}+\mathrm{s}$ stars, but the r-only rich very metalpoor stars are so rare that it is implausible to explain a larger population (of $r+s$ stars) through an even rarer one.

The imbalance of the two populations would even push towards the reverse scenario (Sect. 4.5).

\subsection{The s-process elements in metal-poor stars}

The late onset of s-process elements in metal-poor stars is interpreted, according to Burris et al. (2000), by the delay necessary for the ejection of the s-elements at the end of the evolution of the massive metal-poor AGBs. These AGBs are presumably formed from interstellar gas enriched in $\mathrm{Fe}$ (and possibly r-elements) by the first SN II, and they feed the interstellar gas with s-elements. The s-rich non-binary classical metal-poor stars observed would then be third generation stars, since they contain some Fe and s-elements. In some phases (AGB) the low mass stars are able to build themselves s-process elements (and dredge up them to external layers): they are then intrinsic s-rich stars. But the analyzed stars are not evolved enough to have reached the AGB phase. They could perhaps have suffered a brief and violent mixing at the core helium flash, and some neutron flux exposure, but the models of He-flash do not produce the expected abundance ratios (Weiss et al. 2004).

In the long period binaries, the enhancement in s-elements has a classical interpretation: mass transfer from a more massive companion in its AGB phase. The exceptionally strong enrichment in $\mathrm{C}, \mathrm{N}$, and s-elements is thus explained by a local event. The stars are extrinsic s-rich stars. They may be second generation stars. However a variable radial velocity has not yet been found for all the $\mathrm{r}+\mathrm{s}$ stars. If these stars are found to be non-binaries, they could have been formed from an interstellar gas cloud locally enriched by the winds of nearby AGB stars, and this is an extension of the classical extrinsic enrichment (Busso et al. 2001): it applies to non-binary stars. This explanation of the enrichment may seem somewhat ad hoc, but the scatter of the abundances of the s- and r-elements is large at low metallicities, so large that it implies a clear inhomogeneity of the interstellar matter.

The $\mathrm{r}+\mathrm{s}$ stars not recognized as binaries may also have been initially long period binaries, ceasing to be binaries due to the explosion of the massive companion.

\subsection{Possible origins of the metal-poor $r+s$ enhanced stars}

It has been noted in Sect. 4.3, that a star with a high ratio r-elements/Fe could, by accreting some s-rich matter, become an $\mathrm{r}+\mathrm{s}$ star; however, given that metal-poor stars with very high r-elements/Fe ratios are very rare, this is not a plausible scenario for the formation of a number of $r+s$ stars (AR02).

The interpretation by Tsujimoto \& Shigeyama (2001) of the well observed supernova 1987A predicts that the r-rich ejecta of this rather low mass supernova could nearly fit the abundances of both light metals and r-elements in the $\mathrm{r}+\mathrm{s}$ stars. It is not impossible that a gas cloud, polluted in r-elements by such low-mass (low energy) SNe II, producing r-rich stars, to be later polluted by an AGB companion. If the strong enrichments in s-elements are explained by local events (mass transfer), it is tempting to try to explain (completely or in part) the enhancement in r-elements also by a local enrichment from a companion, in this case producing predominantly r-elements. Some scenarios have been proposed:

(i) For our $\mathrm{r}+\mathrm{s}$ stars, a first scenario invoking a triple system (one component polluting with r-elements, and another polluting with s-elements) may be considered, but is does not seem very likely (CC03), moreover such a triple system may be dynamically not stable.

(ii) In a second scenario, $\mathrm{CC} 03$ proposes that, after mass loss, the polluting massive AGB companion evolves into a white dwarf which accretes a substantial amount of mass from the s-enriched giant star, then collapses to a neutron star (Accretion Induced collapse: AIC), producing r-elements ejected towards the surface of the observed 
star. Some features of this scenario may be considered as drawbacks:

a) some of the superficial s-enriched matter leaves the giant to go back to the evolved companion, weakening the previous enrichment;

b) for an efficient accretion from the giant to the white dwarf, the orbit has to remain a close one, whereas the long period current orbits are not close, but they may have changed (maybe by an explosion);

c) the production of significant amounts of r-elements by the AIC process has not yet been completely proven.

(iii) For a third scenario, Zijlstra (2004) proposes that the primary (evolved as an AGB) transfers s-rich matter to the observed star but does not suffer a large mass loss (owing to its low metallicity). At the end of the AGB phase, the degenerate core is massive enough for exploding as a supernova of type SN 1.5 (so called by Iben \& Renzini 1983) now called an AGB supernova. In this scenario the massloss is a crucial issue. Computations by Herwig (2004) for moderately massive AGBs would indicate a low mass for the final degenerate core, which would then exclude collapse as a SN 1.5. Alternatively, massive AGBs building massive cores could be considered. The evolution of the mass of the core (and especially the mass loss rate) are difficult problems, so that at present the collapse of a massive $\mathrm{AGB}\left(M \approx 10-12 M_{\odot}\right)$ by electron capture is not excluded (Siess, private communication), perhaps producing r-elements. Such a large mass AGB star could possibly provide the observed enhancement of s-process elements in a first phase, and explode or collapse providing the r-elements (Zijlstra, Siess, private communication), but the modelisation of the evolution of such a large mass metal-poor star is a difficult task (especially the estimation of the mass loss).

In all these scenarios, the r-rich mass which would be transferred to the observed star should not alter the high observed ratio of $[\alpha$-elements $/ \mathrm{Fe}]$. It could have a very high ratio [r-elements/Fe] and could even be devoid of Fe, in agreement with the ideas of Qian \& Wasserburg (2003). It could also have about the same high ratio ([ $\alpha$-elements/Fe]) as the observed star. In fact, the observed ratio $[\alpha$-elements/Fe] is particularly high (see Tables 4, and 3 of CC03), even excluding from the computation the proton capture elements like $\mathrm{Na}, \mathrm{Al}$ or possibly $\mathrm{Mg}$, which may have been partly synthesized by deep CNO burning in the massive companion.

Finally, Johnson \& Bolte (2004) have recently analyzed a metal-poor star, similar to the two stars analyzed here. They note that their observations cannot be completely matched by any combination of s-and r-processes, and propose an alternative explanation. They try to interpret the abundance pattern of the heavy elements by theoretical metal-poor s-processes, and although the general trend from $\mathrm{Y}$ to $\mathrm{Pb}$ is somewhat better represented than using the solar process, the abundances of some elements are not satifactorily explained. Further investigations are obviously needed.

\subsection{A possible scenario for the r-only stars?}

A modified scenario could possibly explain the rare and mysterious stars strongly enriched in r-only elements such as CS 22892-052 (Sneden et al. 1996, 2003), and CS 31082-001 (Cayrel et al. 2001; Hill et al. 2002). These stars could be formed in a close orbit version of the scenarios forming the $\mathrm{r}+\mathrm{s}$ stars, favoring an early mass transfer from the massive companion in its RGB phase. Admittedly, no variation of radial velocity has yet been observed for one of these two r-only stars, but the explosion which would have provided the r-elements could have also disrupted the binary system. If the massive companion produces some $\mathrm{C}$ (and $\mathrm{N}$ ) but no s-elements, if its final explosion produces r-elements, this scenario forms, by local enrichment, a r-only rich star, explaining simultaneously the mysterious enrichment in $\mathrm{C}$ (and $\mathrm{N}$ ) sometimes observed in these stars.

Finally, the r-only stars could be something like the $\mathrm{r}+\mathrm{s}$ binaries deprived from s-elements.

Probably other scenarios are possible. As already noted, the interpretation by Tsujimoto \& Shigeyama (2001) of the supernova 1987A, if confirmed, could explain by local enrichment the abundances of both light metals and r-elements in the two known extremely metal-poor r-only rich stars.

\subsection{Some constraints on the scenarios}

The first 4 stars of AR02 in Table 5 are very similar to the two stars analyzed here and the one by $\mathrm{CC} 03$. Two of them have not yet been found to be binaries (AR02, AR03). If they are confirmed as non-binaries, the scenarios based on local inhomogeneities of the star forming gas, or the scenarios including an explosion of the massive companion and the disruption of the binary system would be favored for such cases.

All the scenarios implying a transfer of r-elements from a companion predict the formation of a neutron star (possibly displaced in a wider orbit or ejected).

In the future, it would be useful to gather for each star precise radial velocities all along a complete orbit, to measure its precise proper motions (e.g. by a satellite like Gaia) in order to find the mass of the unseen companion (remnant), the excentricity of the orbit. If the orbital plane is perpendicular to the line of sight, precise proper motions will decide about binarity, and contribute to the definition of the orbit. Interferometric measurements would help. Spectra in the UV could also provide more information about r-elements, and X-ray observations (and even neutrino observations) would be of interest.

Finally, it is interesting to note that no enhancement of the proton capture elements $\mathrm{Na}$ and $\mathrm{Al}$ is observed in field metal poor stars, whereas they are common in globular clusters, and often interpreted as enrichment by the winds of AGB stars, the produced elements being retained in the potential well of the cluster. The two field stars analyzed here show a somewhat similar process. Therefore, enhancement in $\mathrm{Na}$ and $\mathrm{Al}$ may be indicative of pollution by an AGB companion, as a general rule. 


\section{Conclusions}

A revised analysis was carried out of the very metal-poor $\mathrm{CH} / \mathrm{CN}$-strong stars CS 22948-27 and CS 29497-34, including a new derivation of heavy element $(Z>35)$ abundances. The abundance patterns are improved, relative to Paper I, regarding $\alpha$-elements and proton-capture elements, especially for the two crucial elements Eu and $\mathrm{Pb}$.

New radial velocity measurements show that both stars are long period binaries, indicating that mass accretion from the (now faded-out) massive companion occurred at its AGB phase (not RGB), explaining the observed high enhancement of the s-elements including $\mathrm{Pb}$.

The abundance patterns of the elements, in particular of the neutron-capture elements, appear to be similar in CS 22948-27 and CS 29497-34. Moreover the two stars are also very similar in terms of kinematic parameters (Galactic velocities), as demonstrated in Paper I.

The abundance patterns are also very similar to that found in the star HE 2148-1247 analyzed by CC03, probably also a long period variable.

These three $r+s$ stars, together with the first four stars of the list of AR02, have rather similar abundance patterns of heavy elements and, at least from this point of view, may form an homogeneous group. They have presumably the same origin, but two of them are not yet known to be binaries. They are not clearly different from the other progressively less extreme stars analysed by AR01, AR02.

The abundances of the neutron capture elements in CS 22948-27 and CS 29497-34 appear to be partly due to s-process and partly due to r-process, about in the same proportions as the solar ones, similarly to what is found for HE 2148-1247 by CC03. Also, the amount of Eu and Ba in these stars is large, comparable to that found in the Sun.

The s-elements in these stars, very probably accreted from a massive companion in its AGB phase, show a large abundance of $\mathrm{Pb}$, in agreement with the computations of Gallino et al. (1998) who predict that low metallicity AGB produce high $\mathrm{Pb}$ abundances (although the prediction is for low mass AGB at contrast to what is expected here). The dominant isotope should be ${ }^{208} \mathrm{~Pb}$. Observations in the $\mathrm{r}+\mathrm{s}$ stars and the predictions of recent models show only a partial agreement (Fig. 8).

The three extreme $\mathrm{r}+\mathrm{s}$ stars, forming a homogeneous group, show also some overabundance of the proton capture elements $\mathrm{Na}$, and $\mathrm{Al}$, presumably formed by deep CNO burning in the massive AGB companion.

The observed r-elements may have been transferred from the massive companion, if, owing to accretion or small mass loss, its final core is massive enough for producing r-elements induced by its collapse.

The enrichment by transfer of r-rich matter has been such as to preserve the clear enhancement of $\alpha$-elements observed in the three $\mathrm{r}+\mathrm{s}$ stars.

This local enrichment by r-process elements, proposed for our $\mathrm{r}+\mathrm{s}$ stars, could perhaps be tentatively extended to the r-only rich stars in a modified version: the orbit would be closer, the mass transfer from the massive companion would be in its RGB phase, the transfer would bring a limited amount of $\mathrm{C}$ (and N), and no s-elements, the explosion of the massive final core would provide the r-elements and would induce (or not) the disruption of the close binary.

The r-only rich stars show also the classical enhancement of the $\alpha$-elements, but not the strong enhancements of the proton capture elements $\mathrm{Na}$ and $\mathrm{Al}$ found in the $\mathrm{r}+\mathrm{s}$ stars (and attributed to the AGB companion).

Further theoretical work is needed regarding yields of low mass zero metal SN II, as well as of metal-poor massive AGB stars, and also about the final phase of the core of metalpoor high mass AGB stars. The observation of a larger sample of stars is advisable, and careful determinations along the years of the orbit of each binary could provide the mass of the evolved companion (remnant), giving useful constraints on the problem of the r-process site(s) in these binaries.

Acknowledgements. We are indebted to Falk Herwig, Albert Zijlstra and Lionel Siess for helpful comments about possible processes of formation of the $\mathrm{r}+\mathrm{s}$ stars. We acknowledge partial financial support from the Observatoire de Paris, CNPq/CNRS and Fapesp.

\section{References}

Alvarez, R., \& Plez, B. 1998, A\&A, 330, 1109

Aoki, W., Ryan, S. G., Norris, J. E., et al. 2001, ApJ, 561, 346 (AR01)

Aoki, W., Ryan, S. G., Norris, J. E., et al. 2002a, ApJ, 580, 1149 (AR02)

Aoki, W., Ando, H., Honda, S., et al. 2002b, PASJ, 54, 427

Aoki, W., Ryan, S. G., Iwamoto, N., et al. 2003, ApJ, 592, L67 (AR03)

Asplund, M., Gustafsson, B., Kiselman, D., \& Eriksson, K. 1997, A\&A, 318, 521

Ballester, P., Modigliani, A., Boitiquin, O., et al. 2000, the Messenger, 101,31

Barbuy, B., Cayrel, R., Spite, M., et al. 1997, A\&A, 317, L63

Barbuy, B., Perrin, M.-N., Katz, D., et al. 2003, A\&A, 404, 661

Baumüller, D., \& Gehren, T. 1997, A\&A, 325, 1088

Baumüller, D., Butler, K., \& Gehren, T. 1998, A\&A, 338, 637

Beers, T. C., Preston, G. W., \& Shectman, S. A. 1992, AJ, 103, 1987

Biehl, D. 1976, Ph.D. Thesis, University of Kiel

Burris, D. L., Pilachowski, C. A., Armandroff, T. E., et al. 2000, ApJ, 544,302

Busso, M., Gallino, R., \& Wasserburg, G. J. 1999, ARA\&A, 37, 239

Busso, M., Gallino, R., Lambert, D. L., Travaglio, C., \& Smith, V. V. 2001, ApJ, 557, 802

Cayrel, R., Hill, V., Beers, T. C., et al. 2001, Nature, 409, 691

Cayrel, R., Depagne, E., Spite, M., et al. 2004, A\&A, 416, 1117

Christlieb, N. 2003, Rev. Mod. Astr., 16, 191

Cohen, J. G., Christlieb, N., Qian, Y.-Z., \& Wasserburg, G. J. 2003, ApJ, 588, 1082 (CC03)

Depagne, E., Hill, V., Spite, M., et al. 2002, A\&A, 390, 187

Edvardsson, B., Andersen, J., Gustafsson, B., et al. 1993, A\&A, 275, 101

Gallino, R., Arlandini, C., Busso, M., et al. 1998, ApJ, 497, 388

Goriely, S., \& Mowlavi, N. 2000, A\&A, 362, 599

Goriely, S., \& Siess, N. 2001, A\&A, 378, L25

Grevesse, N., \& Sauval, A. J. 1998, SSRev., 35, 161

Gustafsson, B., Bell, R. A., Eriksson, K., \& Nordlund, ^̊. 1975, A\&A, 42,407 
Gustafsson, B., Edvardsson, B., Eriksson, K., et al. 2003, in IAU Symp. 210, ed. I. Hubeny, D. Mihalas, \& K. Werner, ASP Conf. Ser., 288, 331

Herwig, F. 2004, ApJS, 155, in press

Hill, V., Barbuy, B., Spite, M., et al. 2000, A\&A, 353, 557 (Paper I)

Hill, V., Plez, B., Cayrel, R., et al. 2002, A\&A, 387, 560

Honda, S., Aoki, W., Kajino, T., et al. 2004, ApJ, 607, 474

Iben, I. Jr., \& Renzini, A. 1983, ARA\&A, 21, 271

Johnson, J. A., \& Bolte, M. 2004, ApJ, 605, 462

Lawler, J. E., Wickliffe, M. E., den Hartog, E. A., \& Sneden, C. 2001a, ApJ, 563, 1075

Lawler, J. E., Bonvallet, G., \& Sneden, C. 2001b, ApJ, 556, 452

Luque, J., \& Crosley, D. R. 1999, SRI Int. Rep., MP 99-009

Lucatello, S., Gratton, R., Cohen, J. G., et al. 2003, ApJ, 125, 875

McWilliam, A. 1998, AJ, 115, 1640

McWilliam, A., Preston, G. W., Sneden, C., \& Searle, L. 1995, AJ, 109,27

Norris, J. E., Ryan, S. G., \& Beers, T. C. 1997, ApJ, 488, 350

Norris, J. E., Ryan, S. G., \& Beers, T. C. 2001, ApJ, 561, 1034

Plez, B., Brett, J. M., \& Nordlund 1992, A\&A, 256, 551

Preston, G. W., \& Sneden, C. 2001, AJ, 122, 1545
Qian, Y.-Z., \& Wasserburg, G. J. 2003, ApJ, 588, 1099

Rossi, S., Beers, T. C., \& Sneden, C. 1999, Third Stromlo Symp., ed. B. K. Gibson, T. S. Axelrod, \& M. E. Putman, ASP Conf., 165, 264

Sivarani, T., Bonifacio, P., Molaro, P., et al. 2004, A\&A, 413, 1073

Sneden, C., McWilliam, A., Preston, G. W., et al. 1996, ApJ, 467, 819

Sneden, C., Cowan, J. J., Lawler, J. E., et al. 2003, ApJ, 591, 936

Travaglio, C., Gallino, R., Busso, M., \& Gratton, R. 2001, ApJ, 549, 346

Travaglio, C., Gallino, R., Arnone, E., et al. 2004, ApJ, 601, 864

Tsujimoto, T., \& Shigeyama, T. 2001, ApJ, 561, L97

Umeda, H., \& Nomoto, K. 2003 [arXiv: astro-ph/0308029]

Van Eck, S., Goriely, S., Jorissen, A., \& Plez, B. 2001, Nature, 412, 793

Van Eck, S., Goriely, S., Jorissen, A., \& Plez, B. 2003, A\&A, 404, 291

Weiss, A., Schlattl, H., Salaris, M., \& Cassisi, S. 2004, A\&A, 422, 217

Wichmann, R., Schmitt, J. H. M. M., \& Hubrig, S. 2003, A\&A, 400, 293

Woosley, S. E., \& Weaver, T. A. 1995, ApJS, 101, 181

Zijlstra, A. A. 2004, MNRAS, 348, L23 